\title{
Oscillation of Nonlinear Impulsive Delay Hyperbolic Equation with Functional Arguments via Riccati Method
}

\author{
M. Zou, Q.X. Ma, A.P. Liu \\ School of Mathematics and Physics, China University of Geosciences \\ Wuhan, China
}

\begin{abstract}
In this paper we mainly deal with the oscillation problem of nonlinear impulsive hyperbolic equation with functional arguments by using integral averaging method and a generalized Riccati technique. A sufficient condition for oscillation of the solutions of nonlinear impulsive hyperbolic equation with functional arguments is obtained.
\end{abstract}

Keywords-hyperbolic equation; oscillation; Riccati method; impulsive; delay

\section{INTRODUCTION}

The theories of nonlinear partial functional differential equations are applied in many fields. In recent years the research of oscillation to impulsive partial differential problems has caught more and more attention. In this paper, we study the oscillation property of the impulsive delay hyperbolic equation

$$
\begin{aligned}
& \frac{\partial}{\partial t}\left(r(t) \frac{\partial}{\partial t} u(x, t)\right)=a(t) h(u(x, t)) \Delta u(x, t)-\sum_{i=1}^{n} b_{i}(t) h_{i}\left(u\left(x, \tau_{i}(t)\right)\right) \Delta u\left(x, \tau_{i}(t)\right) \\
&+\sum_{j=1}^{m} q_{j}(x, t) \varphi_{j}(u(x, t)), \quad t \neq t_{k}, \quad(x, t) \in \Omega \equiv G \times(0,+\infty), \\
& u\left(x, t_{k}^{+}\right)-u\left(x, t_{k}^{-}\right)=\alpha_{k} u\left(x, t_{k}\right) \quad t=t_{k}, k=1,2, \cdots, \\
& u_{t}\left(x, t_{k}^{+}\right)-u_{t}\left(x, t_{k}^{-}\right)=\beta_{k} u_{t}\left(x, t_{k}\right) \quad t=t_{k}, k=1,2, \cdots,
\end{aligned}
$$

where $G$ is a bounded domain of $\mathbb{R}^{n}$ with the smooth boundary $\partial G$. We consider the following boundary condition:

$$
u=0 \quad(x, t) \in \partial G \times[0,+\infty)
$$

Following are the basic hypotheses:

$$
r(t) \in C([0,+\infty) ;(0,+\infty))
$$

$a(t), b_{i}(t) \in P C([0,+\infty) ;[0,+\infty)), i=1,2, \cdots n$,

$q_{j}(x, t) \in C(\bar{\Omega} ;[0,+\infty)), \quad j=1,2, \cdots, m$, where PC denotes the class of functions which are piecewise continuous in $t$ with discontinuities of the first kind only at $t=t_{k}, k=1,2, \cdots$.

(H2) $\tau_{i}(t) \in C([0,+\infty) ; R), \lim _{t \rightarrow+\infty} \tau_{i}(t)=+\infty, i=1,2, \cdots, n$.

(H3) $\quad h^{\prime}(u), h_{i}^{\prime}(u) \in C(R, R) \quad, \quad \varphi_{j}(s) \in C(R, R)$, $\alpha_{k}, \beta_{k}=$ const. $>-1, u h^{\prime}(u) \geq 0, u h_{i}^{\prime}(u) \geq 0, h(0)=0, h_{i}(0)=0$,

$$
\begin{aligned}
& i=1,2, \cdots, n \quad, \quad \frac{\varphi_{j}(s)}{s} \geq C_{j}=\text { const. }>0 \quad \text { for } \quad s \neq 0 . \\
& 0<t_{1}<t_{2}<\cdots<t_{k}<\cdots, \lim _{t \rightarrow+\infty} t_{k}=+\infty, k=1,2, \cdots .
\end{aligned}
$$

We introduce the notations: $U(t)=\int_{G} u(x, t) d x$ and $q_{j}(t)=\min _{x \in \bar{G}} q_{j}(x, t)$.

Definition 1.1. By a solution $u(x, t)$ of problem (1)-(4) we mean a function $u(x, t) \in C^{2}\left(\bar{G} \times\left[t_{-1}, \infty\right)\right)$ which satisfies problem (1)-(4), where

$$
t_{-1}=\min \left\{0, \min _{1 \leq i \leq n}\left\{\inf _{t \geq 0} \tau_{i}(\mathrm{t})\right\}\right\} .
$$

Definition 1.2. The solution $u(x, t)$ of problem (1)-(4) is said to be non- oscillatory in domain $\Omega$ if it is either eventually positive or eventually negative. Otherwise, it is called oscillatory.

Definition 1.3. We say that functions $\left(H_{1}, H_{2}\right)$ belong to a function class $\mathcal{H}$, denoted by $\left(H_{1}, H_{2}\right) \in \mathcal{H}$, if $\left(H_{1}, H_{2}\right) \in C(D ;[0,+\infty))$ satisfy

$$
H_{i}(t, t)=0, H_{i}(t, s)>0(i=1,2) \text { for } t>s,
$$

where $D=\{(t, s): 0<s \leq t<+\infty\}$. Moreover, the partial derivatives $\partial H_{1} / \partial t$ and $\partial H_{2} / \partial s$ exist on $D$ such that

$$
\frac{\partial H_{1}}{\partial t}(s, t)=h_{1}(s, t) H_{1}(s, t) \text { and } \frac{\partial H_{2}}{\partial s}(t, s)=-h_{2}(t, s) H_{2}(t, s) \text {, }
$$

where $h_{1}, h_{2} \in C_{\text {loc }}(D ; \mathbb{R})$.

In recent years, there has been much research activity concerning the oscillation theory of nonlinear hyperbolic equations with functional arguments by employing Riccati technique. Riccati techniques were used to obtain various oscillation results. Recently, Y.Shoukaku and N. Yoshida [2] derived oscillation criteria by using oscillation criteria of Riccati inequality. In this work, we study the hyperbolic equation with impulsive. 


\section{MAIN RESULTS}

Theorem 2.1. If for each $T \geq 0$, there exist $\left(H_{1}, H_{2}\right) \in \mathcal{H}$ and $a, b, c \in \mathbb{R}$ such that $T \leq a<c<b$ and

$$
\begin{array}{r}
\frac{1}{H_{1}(c, a)} \int_{a}^{c} H_{1}(s, a) \prod_{t_{1} \leq t_{k}<s}\left(\frac{1+\beta_{k}}{1+\alpha_{k}}\right)^{-1}\left(C_{l} q_{l}(s)-\frac{1}{4} r(s) \lambda_{1}^{2}(s, a)\right) \psi(s) d s \\
+\frac{1}{H_{2}(b, c)} \int_{c}^{b} H_{2}(b, s) \prod_{t_{1} \leq t_{k}<s}\left(\frac{1+\beta_{k}}{1+\alpha_{k}}\right)^{-1}\left(C_{l} q_{l}(s)-\frac{1}{4} r(s) \lambda_{2}^{2}(b, s)\right) \psi(s) d s>0,
\end{array}
$$

then (1)-(4) has no eventually positive solution, where $\psi(t) \in C^{1}\left(\left(T_{0},+\infty\right) ;(0,+\infty)\right)$ for some $T_{0}>0$ and

$$
\begin{gathered}
\lambda_{1}(s, t)=\frac{\psi^{\prime}(s)}{\psi(s)}+h_{1}(s, t), \\
\lambda_{2}(t, s)=\frac{\psi^{\prime}(s)}{\psi(s)}-h_{2}(t, s) .
\end{gathered}
$$

Proof. Suppose to the contrary that there is a nonoscillatory solution $u(x, t)$ of the problem (1) - (4). Without loss of generality we may assume that $u(x, t)>0$ in $G \times\left[t_{0},+\infty\right)$ for some $t_{0}>0$ because the case where $u(x, t)<0$ can be treated similarly. Since (H2) holds, we see that $u\left(x, \tau_{i}(t)\right)>0(i=1,2, \cdots n)$ in $G \times\left[t_{1},+\infty\right)$ for some $t_{1} \geq t_{0}$.

(1) For $t \geq t_{1}, t \neq t_{k}, k=1,2, \cdots$, integrating (1) with respect to $x$ over $G$,we obtain

$\frac{d}{d t}\left(r(t) \int_{G} \frac{\partial}{\partial t} u(x, t) d x\right)=a(t) \int_{G} h(u(x, t)) \Delta u(x, t) d x-\sum_{j=1}^{m} \int_{G} q_{j}(x, t) \varphi_{j}(u(x, t)) d x$

$+\sum_{i=1}^{n} b_{i}(t) \int_{G} h_{i}\left(u\left(x, \tau_{i}(t)\right)\right) \Delta u\left(x, \tau_{i}(t)\right) d x$.

By Green's formula and the boundary condition, we have

$\int_{G} h(u(x, t)) \Delta u(x, t) d x=\int_{\partial G} h(u(x, t)) \frac{\partial u(x, t)}{\partial n} d s-\int_{G} h^{\prime}(u)|\operatorname{gradu}|^{2} d x$

$$
=-\int_{G} h^{\prime}(u)|\operatorname{gradu}|^{2} d x \leq 0 .
$$

$\int_{G} h_{i}\left(u\left(x, \tau_{i}(t)\right)\right) \Delta u\left(x, \tau_{i}(t)\right) d x \leq 0$.

For condition (H3) we can easily obtain $\int_{G} q_{j}(x, t) \varphi_{j}(u(x, t)) d x \geq C_{j} q_{j}(t) \int_{G} u(x, t) d x$.

Then $U(t)>0$, and it follows that

$\left(r(t) U^{\prime}(t)\right)^{\prime}+\sum_{j=1}^{m} C_{j}(t) q_{j}(t) U(t) \leq 0$.

For some $l \in\{1,2, \cdots, m\}$, we can get $\left(r(t) U^{\prime}(t)\right)^{\prime}+C_{l} q_{l}(t) U(t) \leq 0, \quad t \geq t_{1}, t \neq t_{k}$.

(2) For $t=t_{k}, k=1,2, \cdots$. From (2)-(3) we have that $\int_{G} u\left(x, t_{k}^{+}\right) d x-\int_{G} u\left(x, t_{k}^{-}\right) d x=\alpha_{k} \int_{G} u\left(x, t_{k}\right)$,
$\int_{G} u_{t}\left(x, t_{k}^{+}\right) d x-\int_{G} u_{t}\left(x, t_{k}^{-}\right) d x=\beta_{k} \int_{G} u_{t}\left(x, t_{k}\right)$,

that is

$U\left(t_{k}^{+}\right)=\left(1+\alpha_{k}\right) U\left(t_{k}\right), U^{\prime}\left(t_{k}^{+}\right)=\left(1+\beta_{k}\right) U^{\prime}\left(t_{k}\right)$.

Thus we obtain that the functions $U(t)$ is a eventually positive solution of the impulsive differential inequality

$$
\left\{\begin{array}{c}
\left(r(t) y^{\prime}(t)\right)^{\prime}+C_{l} q_{l}(t) y(t) \leq 0, \\
y\left(t_{k}^{+}\right)=\left(1+\alpha_{k}\right) y\left(t_{k}\right), \\
y^{\prime}\left(t_{k}^{+}\right)=\left(1+\beta_{k}\right) y^{\prime}\left(t_{k}\right) .
\end{array}\right.
$$

Set $w(t)=\frac{r(t) U^{\prime}(t)}{U(t)}$ for $t \geq t_{1}$. From (6), we obtain that $w^{\prime}(t)+\frac{1}{r(t)} w^{2}(t) \leq-C_{l} q_{l}(t)$

$w\left(t_{k}^{+}\right)=\frac{1+\beta_{k}}{1+\alpha_{k}} w\left(t_{k}\right) \cdot$

Define $v(t)=\prod_{t_{1} \leq t_{k}<t}\left(\frac{1+\beta_{k}}{1+\alpha_{k}}\right)^{-1} w(t)$. In fact, $w(t)$ is continuous on each interval $\left(t_{k}, t_{k+1}\right]$, and in view of $w\left(t_{k}^{+}\right)=\frac{1+\beta_{k}}{1+\alpha_{k}} w\left(t_{k}\right)$, it follows that for $t \geq t_{1}$

$v\left(t_{k}^{+}\right)=\prod_{t_{1} \leq t_{j} \leq t_{k}}\left(\frac{1+\beta_{k}}{1+\alpha_{k}}\right)^{-1} w\left(t_{k}^{+}\right)=\prod_{t_{1} \leq t_{j}<t_{k}}\left(\frac{1+\beta_{k}}{1+\alpha_{k}}\right)^{-1} w\left(t_{k}\right)=v\left(t_{k}\right)$,

and for all $t \geq t_{1}$

$v\left(t_{k}^{-}\right)=\prod_{t_{1} \leq t_{j} \leq t_{k-1}}\left(\frac{1+\beta_{k}}{1+\alpha_{k}}\right)^{-1} w\left(t_{k}^{-}\right)=\prod_{t_{1} \leq t_{j}<t_{k}}\left(\frac{1+\beta_{k}}{1+\alpha_{k}}\right)^{-1} w\left(t_{k}\right)=v\left(t_{k}\right)$,

which implies that $v(t)$ is continuous on $\left[t_{1},+\infty\right)$.

$$
\begin{aligned}
& v^{\prime}(t)+\prod_{t_{1} \leq t_{k}<t} \frac{1+\beta_{k}}{1+\alpha_{k}} \frac{1}{r(t)} v^{2}(t)+\prod_{t_{1} \leq t_{k}<t}\left(\frac{1+\beta_{k}}{1+\alpha_{k}}\right)^{-1} C_{l} q_{l}(t) \\
& =\prod_{t_{1} \leq t_{k}<t}\left(\frac{1+\beta_{k}}{1+\alpha_{k}}\right)^{-1} w^{\prime}(t)+\frac{1}{r(t)} \prod_{t_{1} t_{k}<t} \frac{1+\beta_{k}}{1+\alpha_{k}}\left(\prod_{t_{1} t_{k}<t}\left(\frac{1+\beta_{k}}{1+\alpha_{k}}\right)^{-1}\right)^{2} w^{2}(t)+\prod_{t_{1} \leq t_{k}<t}\left(\frac{1+\beta_{k}}{1+\alpha_{k}}\right)^{-1} C_{l} q_{l}(t) \\
& =\prod_{t_{1} \leq t_{k}<t}\left(\frac{1+\beta_{k}}{1+\alpha_{k}}\right)^{-1}\left[w^{\prime}(t)+\frac{1}{r(t)} w^{2}(t)+C_{l} q_{l}(t)\right] \leq 0 .
\end{aligned}
$$

That is to say

$$
v^{\prime}(t)+\prod_{t_{1} \leq t_{k}<t} \frac{1+\beta_{k}}{1+\alpha_{k}} \frac{1}{r(t)} v^{2}(t) \leq-\prod_{t_{1} \leq t_{k}<t}\left(\frac{1+\beta_{k}}{1+\alpha_{k}}\right)^{-1} C_{l} q_{l}(t) .
$$

Multiplying (7) by $\psi(s)$, we obtain

$$
\prod_{t_{1} \leq t_{k}<s}\left(\frac{1+\beta_{k}}{1+\alpha_{k}}\right)^{-1} \psi(s) C_{l} q_{l}(s) \leq-\psi(s) v^{\prime}(s)-\prod_{t_{1} \leq t_{k}<s} \frac{1+\beta_{k}}{1+\alpha_{k}} \frac{\psi(s)}{r(s)} v^{2}(s) .
$$


Multiplying (8) by $H_{2}(t, s)$ and integrating over $[c, t]$ for $t \in[c, b)$, we have

$\int_{c}^{t} \prod_{t_{1} \leq t_{k}<s}\left(\frac{1+\beta_{k}}{1+\alpha_{k}}\right)^{-1} H_{2}(t, s) \psi(s) C_{l} q_{l}(s) d s$

$\leq-\int_{c}^{t} H_{2}(t, s) \psi(s) v^{\prime}(s) d s-\int_{c}^{t} H_{2}(t, s) \prod_{t_{1} \leq t_{k}<s} \frac{1+\beta_{k}}{1+\alpha_{k}} \frac{\psi(s)}{r(s)} v^{2}(s) d s$

$=H_{2}(t, c) v(c) \psi(c) \quad-\int_{c}^{t} H_{2}(t, s)\left(\sqrt{\frac{\prod_{t t_{k}<s} \frac{1+\beta_{k}}{r(s)}}{r(s)}} v(s)-\frac{1}{2} \lambda_{2}(t, s) \sqrt{\left.\frac{r(s)}{\prod_{t, s_{k}<s} \frac{1+\beta_{k}}{1+\alpha_{k}}}\right)^{2}} \psi(s) d s\right.$

$+\frac{1}{4} \int_{c}^{t} \prod_{t_{1} \leq t_{k}<s}\left(\frac{1+\beta_{k}}{1+\alpha_{k}}\right)^{-1} H_{2}(t, s) r(s) \psi(s) \lambda_{2}^{2}(t, s) d s$

$\leq H_{2}(t, c) v(c) \psi(c)+\frac{1}{4} \int_{c}^{t} \prod_{t_{1} \leq t_{k}<s}\left(\frac{1+\beta_{k}}{1+\alpha_{k}}\right)^{-1} H_{2}(t, s) r(s) \psi(s) \lambda_{2}^{2}(t, s) d s$,

and so

$\frac{1}{H_{2}(t, c)} \int_{c}^{t} H_{2}(t, s)\left(\prod_{t_{1} \leq t_{k}<s}\left(\frac{1+\beta_{k}}{1+\alpha_{k}}\right)^{-1} C_{l} q_{l}(s)-\frac{1}{4} \prod_{t_{1} t_{k}<s}\left(\frac{1+\beta_{k}}{1+\alpha_{k}}\right)^{-1} r(s) \lambda_{2}^{2}(t, s)\right) \psi(s) d s$ $\leq v(c) \psi(c)$.

Letting $t \rightarrow b^{-}$in the above, we obtain

$$
\begin{gathered}
\frac{1}{H_{2}(b, c)} \int_{c}^{b} H_{2}(b, s)\left(\prod_{t_{1} \leq t_{k}<s}\left(\frac{1+\beta_{k}}{1+\alpha_{k}}\right)^{-1} C_{l} q_{l}(s)-\frac{1}{4} \prod_{t_{1} \leq t_{k}<s}\left(\frac{1+\beta_{k}}{1+\alpha_{k}}\right)^{-1} r(s) \lambda_{2}^{2}(b, s)\right) \psi(s) d s \\
\leq v(c) \psi(c) .
\end{gathered}
$$

On the other hand, multiplying (8) by $H_{1}(s, t)$ and integrating over $[t, c]$ for $t \in(a, c]$, we obtain

$\int_{t}^{c} \prod_{t_{1} \leq t_{k}<s}\left(\frac{1+\beta_{k}}{1+\alpha_{k}}\right)^{-1} H_{1}(s, t) \psi(s) C_{l} q_{l}(s) d s$

$\leq-\int_{t}^{c} H_{1}(s, t) \psi(s) v^{\prime}(s) d s-\int_{t}^{c} H_{1}(s, t) \prod_{t_{1} \leq t_{k}<s} \frac{1+\beta_{k}}{1+\alpha_{k}} \frac{\psi(s)}{r(s)} v^{2}(s) d s$

$=-H_{1}(c, t) v(c) \psi(c)$

$-\int_{t}^{c} H_{1}(s, t)\left(\sqrt{\frac{\prod_{t_{1} t_{k}<s} \frac{1+\beta_{k}}{r(s)}}{r(s)}} v(s)-\frac{1}{2} \lambda_{1}(s, t) \sqrt{\frac{r(s)}{\prod_{t_{1} s_{k}<s} \frac{1+\beta_{k}}{1+\alpha_{k}}}}\right)^{2} \psi(s) d s$

$+\frac{1}{4} \int_{t}^{c} \prod_{t_{1} \leq t_{k}<s}\left(\frac{1+\beta_{k}}{1+\alpha_{k}}\right)^{-1} H_{1}(s, t) r(s) \psi(s) \lambda_{1}^{2}(s, t) d s$

$\leq-H_{1}(c, t) v(c) \psi(c)+\frac{1}{4} \int_{t}^{c} \prod_{t_{1} \leq t_{k}<s}\left(\frac{1+\beta_{k}}{1+\alpha_{k}}\right)^{-1} H_{1}(s, t) r(s) \psi(s) \lambda_{1}^{2}(s, t) d s$,

and so

$\frac{1}{H_{1}(c, t)} \int_{t}^{c} H_{1}(s, t)\left(\prod_{t_{1} \leq t_{k}<s}\left(\frac{1+\beta_{k}}{1+\alpha_{k}}\right)^{-1} C_{l} q_{l}(s)-\frac{1}{4} \prod_{t_{1} \leq t_{k}<s}\left(\frac{1+\beta_{k}}{1+\alpha_{k}}\right)^{-1} r(s) \lambda_{1}^{2}(s, t)\right) \psi(s) d s$ $\leq-v(c) \psi(c)$.

Letting $t \rightarrow a^{+}$in the above, we get

$$
\begin{gathered}
\frac{1}{H_{1}(c, a)} \int_{a}^{c} H_{1}(s, a)\left(\prod_{t_{1} \leq l_{k}<s}\left(\frac{1+\beta_{k}}{1+\alpha_{k}}\right)^{-1} C_{l} q_{l}(s)-\frac{1}{4} \prod_{t_{1} \leq l_{k}<s}\left(\frac{1+\beta_{k}}{1+\alpha_{k}}\right)^{-1} r(s) \lambda_{1}^{2}(s, a)\right) \psi(s) d s \\
\leq-v(c) \psi(c) .
\end{gathered}
$$

Adding (9) and (10), we easily obtain the following:

$$
\begin{aligned}
& \frac{1}{H_{1}(c, a)} \int_{a}^{c} H_{1}(s, a) \prod_{t_{1} \leq t_{k}<s}\left(\frac{1+\beta_{k}}{1+\alpha_{k}}\right)^{-1}\left(C_{l} q_{l}(s)-\frac{1}{4} r(s) \lambda_{1}^{2}(s, a)\right) \psi(s) d s \\
& +\frac{1}{H_{2}(b, c)} \int_{c}^{b} H_{2}(b, s) \prod_{t_{1} \leq t_{k}<s}\left(\frac{1+\beta_{k}}{1+\alpha_{k}}\right)^{-1}\left(C_{l} q_{l}(s)-\frac{1}{4} r(s) \lambda_{2}^{2}(b, s)\right) \psi(s) d s \leq 0,
\end{aligned}
$$

which contradicts the condition (5).

\section{ACKNOWLEDGEMENTS}

The authors express their sincere thanks to the referee for valuable suggestions. The Project was Supported by the Fundamental Research Funds for the Central Universities, China University of Geosciences(Wuhan)No. 2012129100 and National Natural Science Foundation of China No. 11201436.

\section{REFERENCES}

[1] V.Lakshmikantham, D.Bainov \& P.S.Simeonov, Theory of impulsive differential equations, World Scientific, Singapore, 1989, 32-33.

[2] Yutaka Shoukaku \& Norio Yoshida, Oscillations of nonlinear hyperbolic equations with functional arguments via Riccati method, Applied Mathematics and Computation, 217(2010), 143-151.

[3] Zhiguo Luo \& Jianhua Shen , Oscillations of second linear differential equations with impulses, Applied Mathematics Letters, 20(2007), 75-81.

[4] D.D.Bainov \& E.Minchev, Oscillation of the solutions of impulsive parabolic equations, Journal of Computational and Applied Mathematics, 69(1996), 207-214

[5] Anping Liu, Ting Liu \& Min Zou, Oscillation of nonlinear impulsive parabolic differential equations of neutral type, Rocky Mountain Journal of Mathematics, (41)2001, 833-850. 